FUT2 GENE ASSOCIATION

$\begin{array}{lrrrr}\text { ИССЛЕДОВАНИЕ } & \text { АССОЦИАЦИИ } & \text { ГЕНА } & \text { FUT2 } & \text { С } \\ \text { ИНФИЦИРОВАННОСТЬЮ } & \text { HЕLІСОВАСТЕR } & \text { PYLORI } & \text { И } \\ \text { КЛИНИЧЕСКИМИ ПРОЯВЛЕНИЯМИ ИНФЕКЦИИ } & & \end{array}$

Шрайнер Е.В. ${ }^{1,2}$

Хавкин А.И.

Kox H. B. ${ }^{1,2}$

Климова А.C ${ }^{2}$

Лифшиц Г. И. ${ }^{1,2}$

${ }^{1}$ Институт химической биологии и фундаментальной медицины

${ }^{2}$ Новосибирский государственный университет

3 Научно-исследовательский клинический институт педиатрии имени академика Ю.Е.Вельтищева Российского национального исследовательского медицинского университета им. Н.И.Пирогова Министерства здравоохранения РФ 4 медицинский институт Белгородского государственного исследовательского университета

\title{
EXAMINING AN ASSOCIATION BETWEEN FUT2 GENE AND HELICOBACTER PYLORI INFECTION RELATED TO CLINICAL MANIFESTATIONS
}

Shrayner E V.a, , 
FUT2 GENE ASSOCIATION

Havkin A.I. ${ }^{\mathrm{c}, \mathrm{d}}$

Kokh N. V. ${ }^{\text {a,b }}$

Klimova A.S. ${ }^{b}$

Lifshits G. I. ${ }^{a, b}$

${ }^{a}$ Institute of Chemical Biology and Fundamental Medicine of the SB RAS

${ }^{\mathrm{b}}$ Novosibirsk state university

${ }^{c}$ The Research and Clinical Institute for Pediatrics named after Academician Yuri

Veltischev of the Pirogov Russian National Research Medical University of the Russian Ministry of Health (Pedklin)

${ }^{\mathrm{d}}$ Institute of medicine, Belgorod State University 
Резюме. Целью исследования было изучение генетического локуса rs602662 FUT2 со статусом инфицированности $H$. pylori и в развитии ассоциированных заболеваний (хронического гастрита, язвенной болезни желудка и 12-перстной кишки).

Методы: В исследование включен 91 пациент, проведено разделение на две группы - «случай» и «контроль». Критерии включения в группу «случай»: диагноз «язвенная болезнь желудка или язвенная болезнь двенадцатиперстной кишки», «хронический неатрофический гастрит»; положительный статус H.pylori. В группу контроля вошли пациенты, с эпизодическими жалобами на диспепсию при прохождении комплексного обследования, имеющие отрицательный статус H. pylori, а также не проходившие курсов элиминации $H$. pylori в анамнезе.

Исследование на наличие полиморфного локуса rs602662 гена FUT2 проводили стандартным методом ПЦР TaqMan на амплификаторе” Real-Time CFX96 Touch”. Продолжительность исследования составила 6 месяцев.

Результаты: В основную группу включено 50 пациентов в возрасте от 21 до 50 лет, в контрольную группу вошел 41 пациент. Пациенты, инфицированные H.pylori чаще отмечали симптомы диспепсии - в $36 \%$, по сравнению с группой контроля -9,7\%. Наличие семейного анамнеза в основной группе по ассоциированным заболеваниям имело значимое отличие, $\chi^{2}=4,97, \mathrm{p}<0,05$.

Для оценки вклада генотипа локуса rs602662 гена FUT2 в риск возникновения клинических проявлений при инфицированности $H$. pylori основная группа была разделена на подгруппы. В распределении аллелей в данных группах выявлены статистически значимые различия. Аллель «А» имеет протективное действие в отношении возникновения клинических симптомов диспепсии. Отношение шансов (OR) при носительстве аллеля «А» (генотипы 
FUT2 GENE ASSOCIATION

A/A и G/A против $\mathrm{G} / \mathrm{G}$ ) иметь клинические симптомы при положительном статусе $H$. pylori составило 0.175 (C.I. $=[0.049-0.625]$ chi2 $=7.79 \mathrm{p}=0.0053$ ).

\section{Заключение:}

1. Не выявлено ассоциативной связи аллелей и генотипов локуса rs602662 гена FUT2 со статусом инфицированности H. pylori.

2. Носительстве аллеля «А» имеют значимую ассоциативную связь с отсутствием клинических симптомов у пациентов с положительным статусом инфицированности H. pylori 0.175 (C.I. $=[0.049-0.625]$ chi2 $=7.79$ p=0.0053).

Ключевые слова: Helicobacter pylori, rs602662, FUT2, диспепсия, хронический гастрит, персонализированная медицина.

Abstract. The aim of the study was to examine an association of the rs602662 FUT2 genetic locus with the status of $H$. pylori infection and development of related diseases (chronic gastritis, gastric ulcer and 12 duodenal ulcer).

Methods: The study included 91 patients, divided into two groups - "case" and "control". Criteria for the "case" group enrollment: diagnosis of gastric or duodenal ulcer, chronic non-atrophic gastritis; positive test for H. pylori.

The "control" group included patients with episodic complaints of dyspepsia while undergoing a comprehensive examination, with negative test for $H$. pylori, as well as having no history of former therapy on $H$. pylori elimination.

The study for the presence of the polymorphic locus rs602662 of the FUT2 gene was carried out by the standard TaqMan PCR method on a Real-Time CFX96 Touch amplifier. The follow-up period was 6 months. 
Results: The main group included 50 patients aged 21 to 50 years, the control group - 41 patients. Patients infected with $H$. pylori more often noticed symptoms of dyspepsia - in 36\%, compared with the control group - 9.7\%. A family history of associated diseases in the main group was significantly differed, $\chi^{2}=4.97, \mathrm{p}<0.05$.

To assess the contribution of the rs602662 locus genotype in FUT2 gene to the risk of clinically manifested $H$. pylori infection, the main group was divided into subgroups. In the distribution of alleles in these groups, significant differences were revealed.

Allele "A" has a protective effect regarding the onset of clinical symptoms of dyspepsia. The odds ratio (OR) with the carriage of allele "A" (genotypes A / A and G / A versus G / G) to have clinical symptoms with a positive H. pylori status was 0.175 $(\mathrm{CI}=[0.049-0.625] \operatorname{chi} 2=7.79 \mathrm{p}=0.0053)$.

\section{Conclusion:}

1. No association of alleles and genotypes of the rs602662 locus of the FUT2 gene with the status of $H$. pylori infection was revealed.

2. Carriage of allele "A" have a significant association with the absence of clinical symptoms in patients with a positive status of $\mathrm{H}$. pylori infection, OR 0.175 $(\mathrm{CI}=[0.049-0.625] \operatorname{chi} 2=7.79 \mathrm{p}=0.0053)$.

Key words: Helicobacter pylori, rs602662, FUT2, dyspepsia, chronic gastritis, personalized medicine. 
1 Колонизация Н. pylori вызывает гуморальные и клеточные иммунные ответы со

2 стороны организма человека. Несмотря на перистальтическое движение 3 кишечного тракта и движение химуса, бактерия устанавливает прочное 4 взаимодействие с эпителиальными поверхностями. Прикрепление бактерий на 5 поверхности эпителия представляет собой взаимодействие между молекулами 6 рецептора на поверхности клетки-хозяина и молекулами адгезина, 7 обнаруженными в оболочке бактериальной клетки [2].

8 На начальном этапе после попадания в желудок Н. pylori, быстро двигаясь при 9 помощи жгутиков, преодолевает защитный слой слизи, связывается с клеточной мембраной и колонизирует слизистую оболочку желудка [4]. Для проникновения к своему месту обитания - в слой пристеночной слизи, бактерия связывается с антигеном системы Льюиса (антиген Le-b), используя находящийся на eе поверхности адгезин-связывающий антиген - адгезин babA [9]. Это взаимодействие является первым и важным этапом патогенности, связанной с Н. pylori, при этом штаммы с пониженной экспрессией $b a b A$, по-видимому, являются менее вирулентными [2, 9].

В исследовании Borén T., Falk Р. в 1993г показано, что прикрепление Н. pylori к эпителию желудка зависит от антигенов группы крови. Далее была установлена взаимосвязь между антигенами Льюис и антигенами системы группы крови $\mathrm{ABH}$, которые имеют общий предшественник антиген Н [2]. Льюис-антигены содержатся в эпителиальных клетках желудка и в слизи, защищающей слизистую оболочку. Иммуногистохимический анализ ткани желудочнокишечного тракта с использованием Fut2-нокаутной мышиной модели показал, что экспрессия структуры Leb антигена зависит от фермента $\alpha$-(1,2)фукозилтрансферазы кодируемого геном FUT2 (Галактозид-2-альфа-Lфукозилтрансфераза 2), и идентифицировал муцин MUC5AC (Mucin 5AC, Oligomeric Mucus/Gel-Forming) в качестве молекулы-носителя структуры Le-b [8]. 


\section{FUT2 GENE ASSOCIATION}

Существуют три варианта антигенов системы Льюиса Le-a и Le-c Le-b, их разнообразие связано с вариантом активности фермента FUT2. Патогенез инфицирования $\mathrm{H}$. pylori и дальнейшего повреждения слизистой оболочки желудка, связан с взаимодействием BabA-адгезина именно с антигеном Le-b.

Ген FUT2, расположен на хромосоме 19. В гене существуют функциональные аллели, влияющие на активность кодируемого фермента, в результате чего выделяют «секреторов» и «несекреторов» FUT2. Вариант rs602662 влияет на статус FUT2 и секрецию Льюис антигенов в эпителиальных клетках желез ЖКТ, секреторным является аллель «G», а аллель «А»- не функциональный. Генотип A/A rs602662 связан с появлением антигенов Le-a и Le-c, генотипы G/A и G/Gантиген Le-b. [2, 9].

В целом заражение Н. pylori зависит от многих факторов среды: вирулентности, состояние слизистой оболочки, сопутствующих заболеваний, генетических факторов - последние изучены мало и представляют интерес в отношении возможности развития методов персонализированной медицины.

Цель данной работы заключается в исследовании ассоциации генетического локуса rs602662 FUT2 со статусом инфицированности Н. pylori и в развитии ассоциированных заболеваний (хронического гастрита, язвенной болезни желудка и 12-перстной кишки).

\section{Методы и материалы}

В исследование включены 91 пациента клиники отдела ЦНМТ ИХБФМ СО РАН, Новосибирск, обратившиеся к гастроэнтерологу на прием. Протокол исследования был одобрено локальным этическим комитетом ЦНМТ (Протокол №01 от 12.10.2018 года). Перед началом анкетирования пациент был осведомлен о целях и задачах исследования, о роли его в данном исследовании. В исследовании использовалась индивидуальная анкета, составленная на основе объективных данных осмотра, данных анамнеза пациента и его генетической 
FUT2 GENE ASSOCIATION

предрасположенности к язвенной болезни желудка и двенадцатиперстной кишки, раку желудка.

Критериями включения являются: возраст от 18 до 50 лет; подписанное информированное согласие; наличие данных исследований: кал на антиген к Helicobacter pylori, видеогастродуоденоскопия (ВГДС), данных «гастропанели».

Критерии исключения: возраст $<18$ лет и $>50$ лет; злоупотребление алкоголем или наркотическими средствами; злоупотребление НПВС в анамнезе; декомпенсация хронических заболеваний, ВИЧ.

Пациенты разделены на две группы.

Критерии включения в группу «случай»: диагноз «язвенная болезнь желудка или язвенная болезнь двенадцатиперстной кишки», «хронический неатрофический гастрит»; положительный статус H.pylori (кал на антиген Нр или результаты цитологии ВГДС).

В группу контроля вошли пациенты, с эпизодическими жалобами на диспепсию при прохождении комплексного обследования, имеющие отрицательный статус антигена к Н. pylori в кале и IgG в крови, а также не проходившие курсов элиминации Н. pylori в анамнезе.

Исследование на наличие полиморфного локуса rs602662 гена FUT2 проводили стандартным методом ПЦР TaqMan на амплификаторе" Real-Time CFX96 Touch" в лаборатории фармакогеномики ИХБФМ СО РАН. Результаты проведённой ПЦР были интерпретированы с помощью программного обеспечения" CFX Maestro для расширенного статистического анализа данных".

Статистическая обработка данных

Результаты исследования обрабатывались с использованием стандартного пакета программ Statistica 8.0. Для данных с непараметрическим распределением рассчитывали М - медиану и 25-й и 75-й квартили (Q25:Q75). 
FUT2 GENE ASSOCIATION

Значимость различий качественных характеристик оценивали с использованием критерия $\chi^{2}$ и одностороннего точного критерия Фишера. Сравнение двух независимых групп по признаку с непараметрическим распределением проводили методом Манна-Уитни.

Расчет соблюдения равновесия Харди-Вайнберга и соотношения шансов (OR) производился с помощью на сайте Института генетики человека (Мюнхен, Германия; https://hg5.helmholtz-muenchen.de/cgi-bin/hw/hwa1.pl) с помощью программы DeFinetti. Данные считались статистически значимыми при рдостигнутый уровень значимости $<0.05$.

\section{Результаты и обсуждение}

В основную группу включено 50 пациентов в возрасте от 21 до 50 лет, в контрольную группу вошел 41 пациент в возрасте от 21 до 50 лет. Медиана возраста в основной группе 31 год, в контрольной 35 лет. По полу в основной группе женщин было 76\% из 50 больных, в контрольной 78\% из 41 больных.

Наличие жалоб на момент анкетирования в группах «случай» и «контроль» существенно отличалась: из 50 пациентов с наличием инфицированности $\mathrm{H}$. pylori симптомы диспепсии отмечали $36 \%$ пациентов (18/50); в группе контроля данный синдром был у отмечен у 9,7\% пациентов (4/41). Закономерно наличие связи между инфицированностью $\mathrm{H}$. pylori и развитием клинической симптоматики - уровень статистической значимости различий $\left(\chi^{2} \mathrm{c}\right.$ поправкой Йетса $=7,08, \mathrm{p}<0.01)$.

В группе «случай» из 18 пациентов постпрандиальный дистресс-синдром (ППДС) был у 10 человек, синдром боли в эпигастрии (СБЭ) у 8 человек; в группе контроля ППДС отмечен у 1 человека, СБЭ у 3 человек. По ППДС между группами уровень статистической значимости различий (точный двусторонний критерий Фишера равен $0,01094, \mathrm{p}<0.05$ ), в отличие от пациентов с СБЭ (точный двусторонний критерий Фишера равен $0,333, \mathrm{p}>0.05)$. Для установления более 
FUT2 GENE ASSOCIATION

точной корреляции требуются группы с включением большего количества человек.

Наличие семейного анамнеза в группах показало уровень статистической значимости различий: в основной группе 16 человек $(32 \%)$ из 50 больных отмечали, что у ближайших кровных родственников в анамнезе есть рак желудка или язвенная болезнь желудка/двенадцатиперстной кишки; в группе контроля данные наследственности были отмечены у 5 пациентов (12\%) из 4, уровень статистической значимости различий $\left(\chi^{2}=4,97, \mathrm{p}<0,05\right)$.

Группы не отличались по количеству курильщиков и пациентов отмечавших выраженное воздействие стрессовых факторов. Положительный статус курения отмечен у 1 пациента из 50 в основной группе и у 2 пациентов из 41 пациента в группе контроля ( $\chi^{2}$ с поправкой Йетса $\left.=0,09, \mathrm{p}>0.5\right)$. Выраженный стресс отмечали 4 пациента в основной группе и 2 пациента из группы контроля $\left(\chi^{2} \mathrm{c}\right.$ поправкой Йетса=0,15, p>0.5).

По результатам анамнестического анализа выявлено, что у пациентов с H.pylori уровень статистической значимости различий отмечается по отягощенной наследственности и клиническим проявлениям, что согласуется с известным в настоящее время постулатом о том, что заражение бактерией происходит внутри семьи. В отношении клинических проявлений диспепсии на сегодняшний день нет четкой связи клинических проявлений и бактерии H.pylori. В то время как полиморфизм гена GN- $\beta 3$ CC может нарушать чувствительность рецепторов к нейротрансмиттерам, стимулирующим двигательную функцию желудка и опорожнение желудка [1]

Для оценки ассоциации генотипа локуса rs602662 гена FUT2 со статусом инфицированности Н. pylori было проведено генотипирование образцов ДНК пациентов из основной группы и группы контроля. Распределение частот генотипов соответствовало закону Харди-Вайнберга (Табл. 1). 


\section{FUT2 GENE ASSOCIATION}

136 Табл. 1. Частоты встречаемости аллелей и генотипов полиморфного локуса 137 rs602662 гена FUT2 в группах «случай» и «контроль».

По изложенным результатам не наблюдается ассоциативной связи генотипов и аллелей гена FUT2 (G/G, G/A, A/A) с инфицированностью Н. pylori. Не выявлен уровень статистической значимости различий между распределением генотипов и аллелей в выборках основной и контрольной групп.

Отношение шансов (OR) иметь положительный статус инфицированности $\mathrm{H}$. pylori у носителей аллеля «G» локуса rs602662 гена FUT2 составило 1.053, C.I. $=[0.567-1.953]$, chi2 $=0.03, \mathrm{p}=0.87$. Полиморфизм rs602662 гена FUT2 не является прогностическими для $\mathrm{H}$. pylori-позитивной серологии в нашем исследовании.

В работе, выполненной при исследовании пациентов жителей Португалии [9] генотип, ассоциированный с функциональным вариантом FUT2 был фактором риска как статуса инфицированности Н. Pylori, так и более выраженными клиническими проявлениями.

В другом исследовании получены противоположенные результаты об отсутствии связи ассоциации генотипа секретора FUT2 H.pylori [10]. В работе статус секретора определяли по генотипу локуса rs601338 FUT2, который находится в сильном неравновесии по сцеплению с исследуемым нами rs602662.

Для оценки вклада генотипа локуса rs602662 гена FUT2 в риск возникновения клинических проявлений при инфицированности Н. pylori основная группа была разделена на подгруппы «1» и «2». Подгруппа «1» с положительным статусом Н. pylori и наличием клинических проявлений -18 пациентов, подгруппа «2» с положительным статусом Н. pylori без клинических проявлений -32 пациента. Распределение частот генотипов в подгруппах соответствовало закону ХардиВайнберга (табл. 2). 
FUT2 GENE ASSOCIATION

162 Табл. 2. Частоты встречаемости аллелей и генотипов полиморфного локуса rs602662 гена FUT2 в подгруппе «1» (с положительным статусом Н. pylori и наличием клинических проявлений) и подгруппе «2» (с положительным статусом Н. pylori без клинических проявлений).

166

167

168

169

170

В распределении аллелей в данных группах выявлены статистически значимые различия. Аллель «А» имеет протективное действие в отношении возникновения клинических симптомов диспепсии. Отношение шансов (OR) при носительстве аллеля «А» (генотипы A/A и G/A против $\mathrm{G} / \mathrm{G}$ ) иметь клинические симптомы при положительном статусе Н. Pylori составило 0.175 (C.I.=[0.049-0.625] chi2=7.79 $\mathrm{p}=0.0053)$ Мы полагаем, что это можно объяснить тем, что наличие аллеля «А» не препятствует проникновению H. Pylori в организм, но создает неблагоприятные условия для проникновения в слой пристеночной слизи. Таким образом дальнейшая персистенция микроорганизма и появлению клинических симптомов у таких пациентов минимально.

По данным базы частот аллелей и генотипов «Gnomad» (https:/gnomad.broadinstitute.org/) примерно 80\% населения в мире являются носителем функционального аллеля «G» rs602662, при этом существуют различия популяционных частот.

В Восточной Азии 100\% населения являются носителями функционального аллеля «G», что может говорить о высоком проценте инфицированности и распространенности гастроэнтерологических патологий. В Южной Азии и Европейских странах на долю функционального аллеля «G» приходится 72\% и $53 \%$, соответственно [9].

По нашим данным, учитывая, что мы не наблюдали статистически значимых различий частот аллелей rs602662 в группах, процент функционального аллеля «G» составил 66,4\% на основании исследования генотипа 91 пациента. 
FUT2 GENE ASSOCIATION

Секреторный статус FUT2 опосредованно через различия в профиле гликопротеинов влияет по-разному влияет на восприимчивость к ряду инфекций в исследованиях. Так фенотип «секретора» FUT2 (аллель G) ассоциирован с восприимчивостью к вирусным инфекциям, включая грипп и норовирус, а также H. plori [5]. «Несекреторный» фенотип FUT2 (аллель А) ассоциирован с восприимчивостью к бактериальным патогенами, включая Streptococcus pneumoniae, Neisseria meningitidis, Haemophilus influenzae и Salmonella Typhimurium [6]. Эта дихотомия в восприимчивости также отражается на склонности к хроническим многофакторным заболеваниям. Так хронический панкреатит (на основании измерения а-амилазы в крови) был ассоциирован с несекреторным фенотипом [14]. В исследовании полногеномного анализа ассоциаций (GWAs) с уровнем витамина В12 в плазме крови локус rs602662 был топовым SNP $\left(\mathrm{p}=2.83 \times 10^{-20}\right)$. Присутствие аллеля «А» в среднем было связано с увеличением концентрации витамина В12 в плазме на 44.2 пг/мл [13], что объяснялось меньшей частотой мальабсорбции из-за хронических воспалительных заболеваний ЖКТ у носителей аллеля «А».

Выводы:

1. Не выявлено ассоциативной связи аллелей и генотипов локуса rs602662 гена FUT2 со статусом инфицированности Н. pylori.

2. Носительстве аллеля «A» (генотипы A/A и $\mathrm{G} / \mathrm{A}$ против $\mathrm{G} / \mathrm{G}$ ) имеют значимую ассоциативную связь с отсутствием клинических симптомов у пациентов с положительным статусом инфицированности Н. pylori 0.175 (C.I. $=[0.049-0.625]$ chi2=7.79 p=0.0053). Это необходимо учитывать при курации пациентов с данным генотипом, так как они с большей вероятностью будут иметь стертую клиническую картину или не иметь ее совсем, что затруднит выявление H.pylori.

Источник финансирования. работа выполнена в рамках бюджетной темы 
FUT2 GENE ASSOCIATION

215 ИХБФМ СО РАН «Развитие методов персонализированной медицины»

216 Конфликт интересов. Авторы декларируют отсутствие явных и потенциальных

217 конфликтов интересов, связанных с публикацией настоящей статьи. 


\section{ТАБЛИЦЫ}

Табл. 1. Частоты встречаемости аллелей и генотипов полиморфного локуса rs602662 гена FUT2 в группах «случай» и «контроль».

Table 1. Frequencies of alleles and genotypes of the rs602662 polymorphic locus of the FUT2 gene in the case and control groups.

\begin{tabular}{|l|l|l|l|l|l|l|}
\hline Группы & \multicolumn{2}{|l|}{$\begin{array}{l}\text { Частота встречаемости } \\
\text { генотипов (чел (\%)) }\end{array}$} & $\begin{array}{l}\text { Частота } \\
\text { встречаемости } \\
\text { аллеля (чел (\%)) }\end{array}$ & $\begin{array}{l}\text { Соответствие } \\
\text { закону } \\
\text { Харди- } \\
\text { Вайнберга, p } \\
\text { (Pearson) }\end{array}$ \\
\cline { 2 - 6 } & G/G & G/A & A/A & G & A & p=0.72 \\
\hline $\begin{array}{l}\text { Группа } \\
\text { «случай» }\end{array}$ & $23(46)$ & $21(42)$ & $6(12)$ & $67(67)$ & $33(33)$ & \\
\hline $\begin{array}{l}\text { Группа } \\
\text { «контроль» }\end{array}$ & $16(39)$ & $22(53,7)$ & $3(7,3)$ & $54(65,9)$ & $28(34,1)$ & $\mathrm{p}=0.22$ \\
\hline
\end{tabular}


Табл. 2. Частоты встречаемости аллелей и генотипов полиморфного локуса rs602662 гена FUT2 в подгруппе «1» (с положительным статусом H. pylori и наличием клинических проявлений) и подгруппе «2» (с положительным статусом H. pylori без клинических проявлений).

Table 2 Frequencies of alleles and genotypes of the polymorphic locus rs602662 of the FUT2 gene in subgroup "1" (with a positive H. pylori status and the presence of clinical manifestations) and subgroup "2" (with a positive status of $\mathbf{H}$. pylori without clinical manifestations).

\begin{tabular}{|c|c|c|c|c|c|c|}
\hline \multirow[t]{2}{*}{ Подгруппа } & \multicolumn{3}{|c|}{$\begin{array}{l}\text { Частота встречаемости } \\
\text { генотипов (чел (\%)) }\end{array}$} & \multicolumn{2}{|c|}{$\begin{array}{l}\text { Частота } \\
\text { встречаемости } \\
\text { аллеля (чел (\%)) }\end{array}$} & \multirow{2}{*}{$\begin{array}{l}\text { Соответствие } \\
\text { закону Харди- } \\
\text { Вайнберга, p } \\
\text { (Pearson) }\end{array}$} \\
\hline & $\mathrm{G} / \mathrm{G}$ & $\mathrm{G} / \mathrm{A}$ & $\mathrm{A} / \mathrm{A}$ & G & $\mathrm{A}$ & \\
\hline 1 & $\begin{array}{l}13 \\
(72,2)\end{array}$ & $4(22,2)$ & $1(0,6)$ & $\begin{array}{l}30 \\
(83,3)\end{array}$ & $6(16,6)$ & $\mathrm{p}=0.39$ \\
\hline 2 & $\begin{array}{l}10 \\
(31,3)\end{array}$ & $\begin{array}{l}17 \\
(53,1)\end{array}$ & $\begin{array}{l}5 \\
(15,6)\end{array}$ & $\begin{array}{l}38 \\
(57,8)\end{array}$ & $27(42,2)$ & $\mathrm{p}=0.61$ \\
\hline
\end{tabular}




\section{МЕТАДАННЫЕ}

Фамилия, имя, отчество, ученая степень, ученое звание, должность автора, ответственного за дальнейшую переписку с редакцией (на русском и английском языках).

Шрайнер Е.В., врач гастроэнтеролог, педиатр, старший преподаватель кафедры акушерства и гинекологии медицинского факультета Новосибирского Государственного Университета, научный сотрудник института химической биологии и фундаментальной медицины, тел. +79833002205, ORCID https://orcid.org/0000-0003-3606-4068, sch704@icloud.com

Shrayner E V, PhD, gastroenterologist, pediatrician, senior lecturer of the Department of Obstetrics and Gynecology, Medical Faculty of Novosibirsk State University, researcher of the institute of chemical, Institute of Chemical Biology and Fundamental Medicine of the SB RAS, tel. +79833002205, ORCID https://orcid.org/0000-00033606-4068, sch704@icloud.com

- Название учреждения, где работает ответственный автор (в русском и официально принятом английском вариантах).

Новосибирский Государственный Университет

- Институт химической биологии и фундаментальной медицины Сибирского отделения Российской академии наук (ИХБФМ СО РАН) Institution - Novosibirsk State University (630090, Novosibirsk, 1 Pirogova st., Russian Federation)

Institute of Chemical Biology and Fundamental Medicine of the SB RAS (630090, Novosibirsk, 8 Lavrentiev Avenue, Russian Federation) 
- Почтовый адрес для переписки с указанием почтового индекса (на русском и английском языках).

630090 г Новосибирск, пр. Ак. Лаврентьева, 8

630090, Novosibirsk, 8 Lavrentiev Avenue, Russian Federation

- Телефон, факс (с указанием кода страны и города), e-mail.

+7983 300 2205, schreinerev@gmail.com

- Фамилия и инициалы остальных соавторов, их ученые степени, ученые звания, должности

Кох Наталья Викторовна врач генетик, н.с. института химической биологии и фундаментальной медицины СО РАН, Новосибирск, старший преподаватель кафедры клинической биохимии медицинского факультета Новосибирского Государственного Университета Лифшиц Галина Израилевна д.м.н., зав. лаборатории персонализированной медицины института химической биологии и фундаментальной медицины СО РАН проф. кафедры внутренних болезней Новосибирского Государственного Университета.

- Полное название статьи, направляемой в редакцию.

Исследование ассоциации гена FUT2 с инфицированностью Helicobacter pylori и клиническими проявлениями инфекции 
FUT2 GENE ASSOCIATION

- Количество страниц текста- 10 страниц, количество рисунков, количество таблиц -2.

- Указать, для какого раздела журнала предназначена работа: оригинальная.

- Дата отправления работы -16.08.2020. 


\section{ТитУльныЙ ЛИСТ}

Название статьи:

ИССЛЕДОВАНИЕ АССОЦИАЦИИ ГЕНА FUТ2 С ИНФИЦИРОВАННОСТЬЮ HELICOBACTER PYLORI И КЛИНИЧЕСКИМИ ПРОЯВЛЕНИЯМИ ИНФЕКЦИИ

EXAMINING AN ASSOCIATION BETWEEN FUT2 GENE AND HELICOBACTER PYLORI INFECTION RELATED TO CLINICAL MANIFESTATIONS

- Фамилия и инициалы остальных соавторов, их ученые степени, ученые звания, должности. (полностью, на русском и английском языках);

Кох Наталья Викторовна врач генетик, н.с. института химической биологии и фундаментальной медицины СО РАН, Новосибирск, старший преподаватель кафедры клинической биохимии медицинского факультета Новосибирского Государственного Университета

Лифшиц Галина Израилевна д.м.н., зав. лаборатории персонализированной медицины института химической биологии и фундаментальной медицины СО РАН проф. кафедры внутренних болезней Новосибирского Государственного Университета.

Kokh N. V. Geneticists, researcher of the institute of chemical, Institute of Chemical Biology and Fundamental Medicine of the SB RAS, Voronina E.N., PhD, Institute of Chemical Biology and Fundamental Medicine of the SB RAS, ORCID: 00000002-3405-6980, voronina_1@mail.ru

G. I. Lifshits, MD, Head of the Laboratory of Personalized Medicine, Institute of Chemical Biology and Fundamental Medicine of the SB RAS, Professor of the Department of Internal Medicine, NSU, ORCID:0000-0001-9048-7710 g162@mail.ru 


\section{FUT2 GENE ASSOCIATION}

- подразделение и учреждение, в котором выполнялась работа (В случае, если авторами статьи являются сотрудники разных учреждений, то последние нумеруются по порядку, начиная с единицы, и соответствующая цифра размещается после фамилии автора, представляющего данное учреждение. Для маркировки авторов в англоязычной части статьи вместо цифр используются латинские буквы (a, b, c, d и т.д.));

Институт химической биологии и фундаментальной медицины ${ }^{1}$

Новосибирский государственный университет ${ }^{2}$

Institute of Chemical Biology and Fundamental Medicine of the SB RAS

Novosibirsk state university ${ }^{\mathrm{b}}$

- сокращенное название статьи для верхнего колонтитула (не более 35 символов, включая пробелы и знаки препинания) (на русском и английском языках); Ассоциация гена FUT2

- не менее 6 ключевых слов на русском и английском языках (на русском и английском языках);

Ключевые слова: Helicobacter pylori, rs602662, FUT2, диспепсия, хронический гастрит, персонализированная медицина.

Key words: dyspepsia, chronic gastritis, personalized medicine

- адрес для переписки с указанием телефона, номера факса и адреса e-mail +79833002205, schreinerev@gmail.com 


\section{СПИСОК ЛИТЕРАТУРЫ}

1. Ивашкин В.Т., Маев И.В., Шептулин А.А. Лапина Т.Л., Трухманов А.С., Картавенко И.М., Киприанис В.А., Охлобыстина О.3. Клинические рекомендации Российской гастроэнтерологической ассоциации по диагностике и лечению функциональной диспепсии // Рос журн гастроэнтерол гепатол колопроктол. 2017. T.27, №1. С. 50-61. [Ivashkin V.T., Mayev I.V., Sheptulin A.A., Lapina T.L., Trukhmanov A.S., Kartavenko I.M., Kiprianis V.A., Okhlobystina O.Z. Diagnosis and treatment of the functional dyspepsia: clinical guidelines of the Russian Gastroenterological Association. Ross z gastroenterol gepatol koloproktol = Russian journal of Gastroenterology, Gepatology and Coloproctology, 2017, vol. 27, no.1, pp. 50-61.doi: 10.22416/1382-4376-2017-27-1-50-61. (In Russ)]

2. Ansari S., Yamaoka Y. Helicobacter pylori BabA in adaptation for gastric colonization. World J Gastroenterol., 2017, vol. 21, no. 23, p.4158-4169. doi: 10.3748/wjg.v23.i23.4158

3. Azevedo M., Eriksson S., Mendes N., Serpa J., Figueiredo C., Resende L.P., Ruvoën-Clouet N., Haas R., Borén T., Pendu J. L., David L. Infection by Helicobacter pylori expressing the BabA adhesin is influenced by the secretor phenotype. J. Pathol., 2008, vol. 215, no. 3, p.308-316. doi: 10.1002/path.2363

4. Bugaytsova J.A., Chernov Y.A., Gideonsson P., Henriksson S., Mendez M., Sjöström R., Mahdavi J., Shevtsova A., Ilver D., Moonens K., P Quintana$\underline{\text { Hayashi }}$ M., Moskalenko R., Aisenbrey C., Bylund G., Schmidt A., Åberg A., Brännström K., Königer V., Vikström S., Rakhimova L., Hofer A., Ögren J., Liu H., Goldman M. D., Whitmire J.M., Ådén J., Younson J., Kelly C. G, Gilman R. H., Chowdhury A., Mukhopadhyay A. K, Nair G.B., Papadakos K.S., Martinez-Gonzalez B., Sgouras D.N., Engstrand L., Unemo M., Danielsson D., Suerbaum S., Oscarson S., Morozova-Roche L. A., Olofsson A., Gröbner G., Holgersson J., Esberg A., Strömberg N., Landström M., Eldridge A. 


\section{FUT2 GENE ASSOCIATION}

M., Chromy B. A., Hansen L. M., Solnick J. V., Lindén S. K., Haas R., Dubois A., Merrell D. S., Schedin S., Remaut H., Arnqvist A., Berg D. E., Borén T. Adaption of Helicobacter pylori to Chronic Infection and Gastric Disease by $\mathrm{pH}$ Responsive BabA-Mediated Adherence- Cell. Host Microbe, 2017 vol. 8, no. 21, p. 376-389. doi: 10.1016/j.chom.2017.02.013

5. Currier R.L., Payne D.C., Staat M.A. Selvarangan R., Shirley S H., Halasa N., Boom J. A, Englund J. A., Szilagyi P. G, Harrison C. J., Klein E. J., Weinberg G. A., Wikswo M. E., Parashar U., Vinjé J., Morrow A. L. Innate susceptibility to norovirus infections influenced by FUT2 genotype in a United States pediatric population. Clin. Infect. Dis. 2015, vol. 60, p.1631-1638. doi:10.1093/cid/civ165

6. Goto Y., Obata T., Kunisawa J., Sato S., Ivanov I. I., Lamichhane A., Takeyama

N., Kamioka M., Sakamoto M., Matsuki T., Setoyama H., Imaoka A., Uematsu

S., Akira S., Domino S. E., Kulig P., Becher B., Renauld J.-C., Sasakawa

C., Umesaki Y., Benno Y., Kiyono H. Innate lymphoid cells regulate intestinal epithelial cell glycosylation. Science, 2014, vol.345, p. 1254009

doi:10.1126/science.1254009

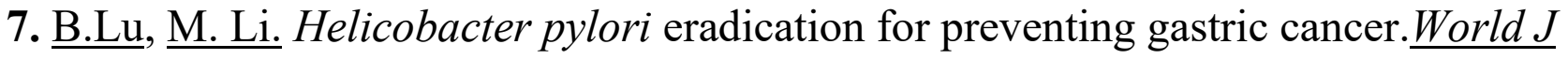
Gastroenterol. 2014; 20(19): 5660-5665. doi: 10.3748/wjg.v20.i19.5660

8. Magalhaes A., Rossez Y., Robbe-Masselot C. Maes E., Gomes J., Shevtsova A., Bugaytsova J., Borén T., Reis C. A. Muc5ac gastric mucin glycosylation is shaped by FUT2 activity and functionally impacts Helicobacter pylori binding. Sci Rep., 2016, vol.6, art.no. 25575. doi:10.1038/srep25575

9. Nell S., Kennemanna L., Schwarz S., Suerbaum S. Dynamics of Lewis b Binding and Sequence Variation of the babA Adhesin Gene during Chronic Helicobacter pylori Infection in Humans. mBio, 2014, vol. 5, no.6, p. e02281-14.

doi:10.1128/mBio.02281-14 


\section{FUT2 GENE ASSOCIATION}

10. Oussalah A., Besseau C., Chery C., Jeannesson E., Guéant-Rodriguez RM., Anello G., Bosco P., Elia M., Romano A., Bronowicki J-P., Gerard P., Paoli J., Avogbe P. H., Chabi N., Sanni A., Amouzou E., Peyrin-Biroulet L., Guéant J-L. Helicobacter pylori serologic status has no influence on the association between fucosyltransferase 2 polymorphism (FUT2 461 G->A) and vitamin B-12 in Europe and West Africa. Am J Clin Nutr, 2012, vol. 95, no.2, p. 514-521. doi: $\underline{10.3945 / a j c n .111 .016410}$

11. Plummer M., Franceschi S., Vignat J., Forman D., de Martel C. Global burden of gastric cancer attributable to Helicobacter pylori. Int J Cancer., 2015, vol.136, p. 487-490. doi:10.1002/ijc.28999

12. Sugano K. , Tack J., Kuipers E.J., Graham D. Y., El-Omar E. M., Miura S., Haruma K., Asaka M., Uemura N., Malfertheiner P. Kyoto global consensus report on Helicobacter pylori gastritis. Gut 2015, vol.64, no.9, p.1353-67. doi: 10.1136/gutjnl-2015-309252

13. Tanaka T., Scheet P., Giusti B., Bandinelli S., Piras M. G., Usala G., Lai S., Mulas A., Corsi A. M., Vestrini A., Sofi F., Gori A. M., Abbate R., Guralnik J., Singleton A., Abecasis G. R., Schlessinger D., Uda M., Ferrucci L. Genome-wide association study of vitamin B6, vitamin B12, folate, and homocysteine blood concentrations. Am J Hum Genet., 2009, vol. 84, no. 4, p.477-482. doi: 10.1016/j.ajhg.2009.02.011

14. Weiss F.U., Schurmann C, Guenther A., Ernst F., Teumer A., Mayerle J., Simon P., Völzke H., Radke D., Greinacher A., Kuehn J-P., Zenker M., Völker U., Homuth G., Lerch M. M. Fucosyltransferase 2 (FUT2) non-secretor status and blood group B are associated with elevated serum lipase activity in asymptomatic subjects, and an increased risk for chronic pancreatitis: a genetic association study. Gut. 2015, vol. 64, p. 646-656. doi: 10.1136/gutjnl-2014-306930 